\title{
Accuracy and Feasibility of a Zero-Setup Implant Guide System Made of a Light-Cured Composite Resin with Simultaneous Flapless Sinus Augmentation: A Pilot Study
}

\author{
Jin-Young Park ${ }^{1,+}+\mathbb{D}$, Joo-Yeon Lee ${ }^{1,+}{ }^{\dagger}$ Joo-Nyeon Kim ${ }^{1}$, Jeong-Won Paik ${ }^{1}$, Jung-Seok Lee ${ }^{1,2}{ }^{\mathbb{D}}$, Ui-Won Jung ${ }^{1}$, \\ Seong-Ho Choi ${ }^{1,2}$ and Jae-Kook Cha ${ }^{1,2, *}$ \\ 1 Department of Periodontology, Research Institute of Periodontal Regeneration, Yonsei University College of \\ Dentistry, Seoul 03722, Korea; jinyoungpark87@gmail.com (J.-Y.P.); dlwndus34@naver.com (J.-Y.L.); \\ wnrxod1021@naver.com (J.-N.K.); jeongwon100@hotmail.com (J.-W.P.); cooldds@gmail.com (J.-S.L.); \\ drjew@yuhs.ac (U.-W.J.); SHCHOI726@yuhs.ac (S.-H.C.) \\ 2 Innovation Research and Support Center for Dental Science, Yonsei University Dental Hospital, \\ Seoul 03722, Korea \\ * Correspondence: chajaekook@gmail.com \\ + These authors contributed equally to the writing of this manuscript.
}

Citation: Park, J.-Y.; Lee, J.-Y.; Kim, J.-N.; Paik, J.-W.; Lee, J.-S.; Jung, U.-W.; Choi, S.-H.; Cha, J.-K. Accuracy and Feasibility of a Zero-Setup Implant Guide System Made of a Light-Cured Composite Resin with Simultaneous Flapless Sinus Augmentation: A Pilot Study. Appl. Sci. 2021, 11, 8085. https:// doi.org/10.3390/app11178085

Academic Editors: Paola Gandini and Andrea Scribante

Received: 12 August 2021

Accepted: 30 August 2021

Published: 31 August 2021

Publisher's Note: MDPI stays neutral with regard to jurisdictional claims in published maps and institutional affiliations.

Copyright: (c) 2021 by the authors. Licensee MDPI, Basel, Switzerland. This article is an open access article distributed under the terms and conditions of the Creative Commons Attribution (CC BY) license (https:/ / creativecommons.org/licenses/by/ $4.0 /)$.

\begin{abstract}
The aim of this study was to evaluate the accuracy and feasibility of flapless implant placement using a light-cured surgical template made of composite resin comprising dimethacrylate and diurethane, performed simultaneously with crestal sinus augmentation. This pilot study was a prospective, single-armed, controlled clinical trial. Four patients having an edentulous region in the posterior maxilla with 4 to $8 \mathrm{~mm}$ of residual ridge height were enrolled. Flapless fully-guided implant placement was performed using a zero-setup surgical template with simultaneous crestal sinus graft. Horizontal, vertical, and angular deviations of the placed implants from the planned positions were measured. Clinical observations were made, and implant stability was measured up to 3 months postoperatively. All four implants were placed and maintained successfully without complication. The mean deviations of the four implants were 1.27 and $1.85 \mathrm{~mm}$ horizontally at platform and apex, respectively, $0.84 \mathrm{~mm}$ vertically at apex, and $4.76^{\circ}$ in angle. Satisfactory implant stability was obtained in all implants at placement and 3 months. Fully-guided implant placement and crestal sinus graft using a zero-setup surgical template can be a safe and feasible method with acceptable accuracy. A randomized controlled trial should be performed in the future to validate its clinical performance in terms of surgical time, accuracy, and patient-reported outcomes.
\end{abstract}

Keywords: guided implant surgery; sinus augmentation; flapless surgery

\section{Introduction}

In the modern era of implant dentistry, it has been well established that the implant fixture should be placed in its prosthetic-driven position [1]. There are many obvious advantages that stem from the correct implant positioning, such as a realistic cervical emergence profile, efficient loading of the occlusal force, and simple oral hygiene [2]. In essence, the correct positioning of the implant fixture determines the long-term survival and success of the implant therapy [3].

Traditionally, implant surgeries were performed free-handed. Precise placement of the implant or several implants into the planned positions required the knowledge and skill of an experienced surgeon [4]. The situation would become even more demanding in a horizontally or vertically atrophic ridge, where guided bone regeneration or maxillary sinus augmentation procedures would be needed. Even an experienced surgeon can be put under pressure in these circumstances, which may result in a severely deviated implant from the initially planned position. 
To compensate for this, various surgical templates have been developed and widely used, which are prefabricated according to a virtual plan devised from computer software using the superimposed data of cone-beam computed tomography (CBCT) and intraoral scanning [5]. These surgical templates can be conveniently fabricated using a 3D printer. The clinical use of the fully-guided surgical templates has many benefits. Apart from the obvious accuracy of implant placement [6], dangerous anatomical structures can be evaded, and an immediate provisional restoration can be delivered [7,8]. Furthermore, a previous study has revealed that the use of a fully-guided surgical template can improve the patient-reported outcome measurements and allow less experienced surgeons to place implants accurately $[9,10]$. However, there is still room for improvement for the existing guide systems. For instance, the guide fabrication process can be time-consuming due to the transfer of data between facilities, and the various steps in the process may create an amplification of errors that may become significant [6].

Recently, a novel, fully-guided surgical template has been proposed, namely the VARO Guide $^{\circledR}$. A unique intraoral device called the "pre-guide" is used to create the VARO guide, which is a preliminary version of the surgical template. The pre-guide looks like a singlesextant bite impression tray and contains light-curable composite resin. The impression of the edentulous sextant is taken with the pre-guide by the curing of the resin, and then CBCT is taken with the pre-guide in situ. The drilling hole locations within the pre-guide are virtually planned on the CBCT imaging. This data is sent to a milling machine that fabricates the fully-guided surgical template from the pre-guide. In summary, the current system can be made inside a single facility (in-house), does not involve a model, be it conventional or digital (model-free), and requires just a milling machine and no laboratory work or a 3D printer (zero-setup). In our previous in vitro study using the VARO guide, a similar placement accuracy was shown compared to a conventional 3D-printed surgical guide; however, the preparation time was much shorter [11]. Now, there is a need to verify the feasibility and accuracy of the VARO guide clinically. In addition, there are only a few studies that have evaluated the accuracy of fully-guided implant placement in the atrophic alveolar ridge [6]. The VARO guide is compatible with a crestal sinus grafting kit that utilizes hydraulic pressure to lift the sinus membrane, which can help to reduce surgical difficulty.

The aim of the present pilot study was to evaluate the accuracy and feasibility of implant placement in the vertically atrophic posterior maxilla using the VARO guide, performed simultaneously with flapless crestal sinus augmentation.

\section{Materials and Methods}

\subsection{Study Design and Patient Selection}

The present pilot study was designed as a prospective, single-armed, controlled clinical trial. All procedures adhered to the Declaration of Helsinki. The trial received ethical approval from the Institutional Review Board of the Yonsei University Dental Hospital (2-2020-0053), and it was registered with the Clinical Research Information Service of the National Research Institute of Health in the Republic of Korea (KCT0005465). Four patients were included in the study who attended the Department of periodontology, Yonsei University Dental Hospital, from 2020 to 2021. Written informed consent was provided by all patients prior to enrollment after being fully informed about the procedures. The procedures were performed by two experienced surgeons who specialized in periodontal and implant therapies.

\subsubsection{Inclusion Criteria}

The inclusion criteria were: (i) being $>19$ years of age, (ii) having a partially edentulous region in the posterior maxilla needing dental implant therapy, (iii) having at least 3 months of healing after the tooth extraction, and (iv) having 4-8 $\mathrm{mm}$ of residual ridge height, allowing sinus augmentation by crestal approach 


\subsubsection{Exclusion Criteria}

A patient satisfying any one of the following exclusion criteria would be excluded from the study: (i) having insufficient residual ridge height and width, which necessitates the implementation of lateral bone augmentation or guided bone regeneration, (ii) containing any lesions in the maxillary sinus including cysts, (iii) having uncontrolled diabetes, (iv) being pregnant or lactating, (v) having a history of radiotherapy or chemotherapy in the head and neck region, (vi) taken bisphosphonates within the past 4 months, (vii) having any other contraindicative oral or systemic diseases to dental implants, and (viii) being a smoker of $>20$ cigarettes a day.

\subsection{Treatment Protocol}

\subsubsection{VARO Guide Fabrication}

The fabrication of the VARO guide (Neobiotech, Seoul, Korea) is illustrated in Figure 1.
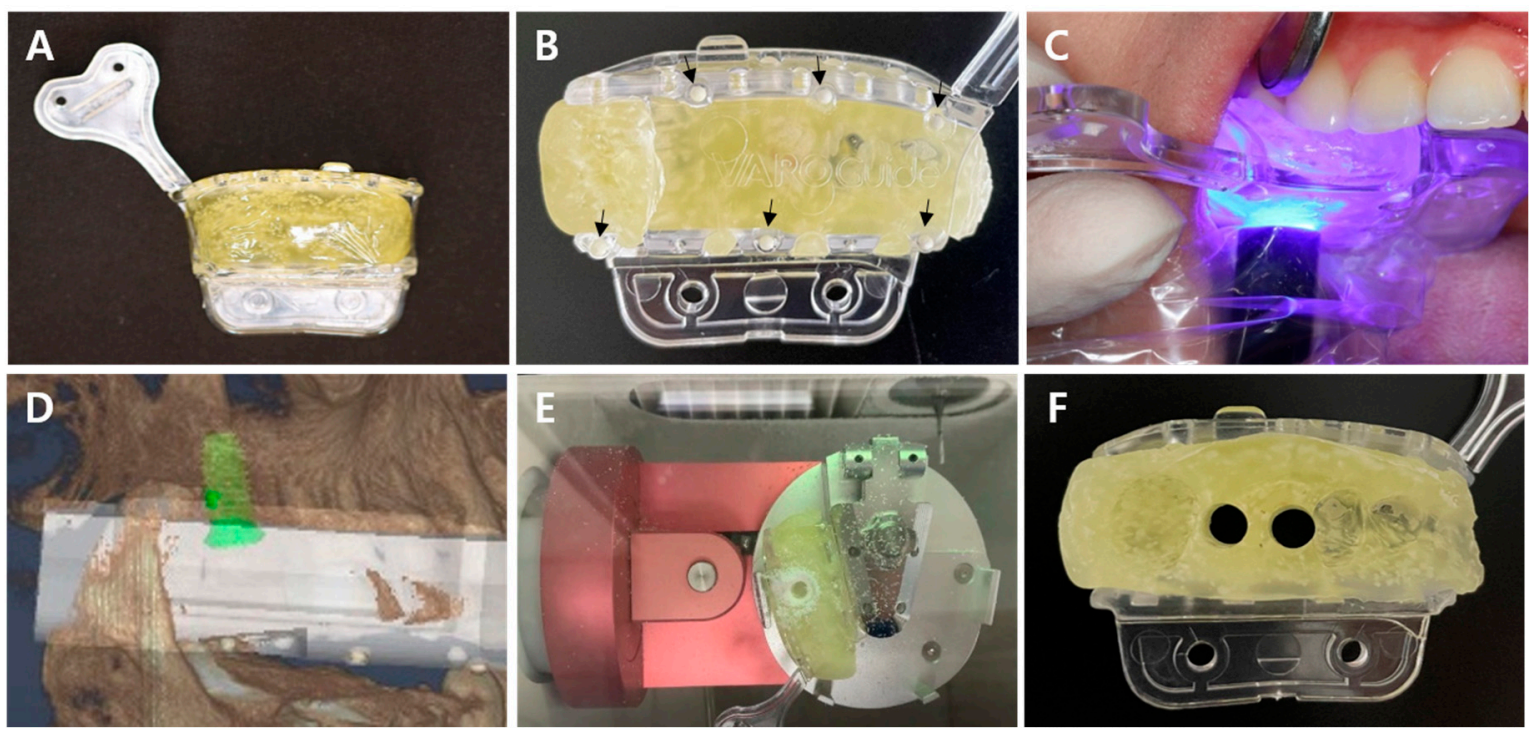

Figure 1. The fabrication process of the VARO guide. (A) A "pre-guide" containing composite resin was opened from its package. (B) The base of the pre-guide containing six radiopaque dots (arrows), which can be recognized by the milling machine. (C) The pre-guide was applied to the edentulous site and then light-cured. (D) Once the cone-beam computed tomography was taken with pre-guide in situ, virtual planning was conducted on computer software. (E) Using the planned data, the VARO guide was fabricated by the VARO milling machine. (F) The completed VARO guide having a drill sleeve.

The fabrication process of the VARO guide is described in detail in a previous study [11]. Following the initial visit of subject enrollment, the VARO guide was fabricated as follows. The pre-guide (PGM13; Neobiotech, Seoul, Korea), containing uncured composite resin comprising dimethacrylate and diurethane was seated on to the posterior maxillary teeth, including the edentulous site. The pre-guide contains a sheet of polyvinyl alcohol that covers the surface of the composite resin, which is intended to prevent the formation of any undercuts during the indentation. With the pre-guide in situ, the composite resin was light-cured from the buccal and palatal sides for $15 \mathrm{~s}$ each. The pre-guide was then retrieved from the mouth, and the biting surfaces were cured for an additional $30 \mathrm{~s}$. Once cured, the pre-guide was repositioned in the mouth and CBCT (Q-FACE, HDX WILL, Seoul, South Korea) was taken ( $85 \mathrm{kV}, 8 \mathrm{~mA}$, and exposure time of $24 \mathrm{~s})$ for both upper and lower jaws. The CBCT images were then transferred to a computer software (VARO Plan; Neobiotech, Seoul, Korea). Pre-surgical planning was performed on the software, and the fixtures were virtually placed in their ideal positions. Drilling holes were subsequently designed according to the fixture locations. This design file was extracted and transferred to a milling machine (VARO Mill; Neobiotech, Seoul, Korea). The pre-guide contains six radio-opaque reference points recognizable by the milling machine, which 
allows precise matching of the planned data with the actual pre-guide. The drilling holes were created in the pre-guide by the milling machine, and the pre-guide is converted to the VARO guide.

\subsubsection{Fully-Guided Implant Placement with Flapless Crestal Sinus Augmentation}

The surgical procedures are summarized in Figure 2.
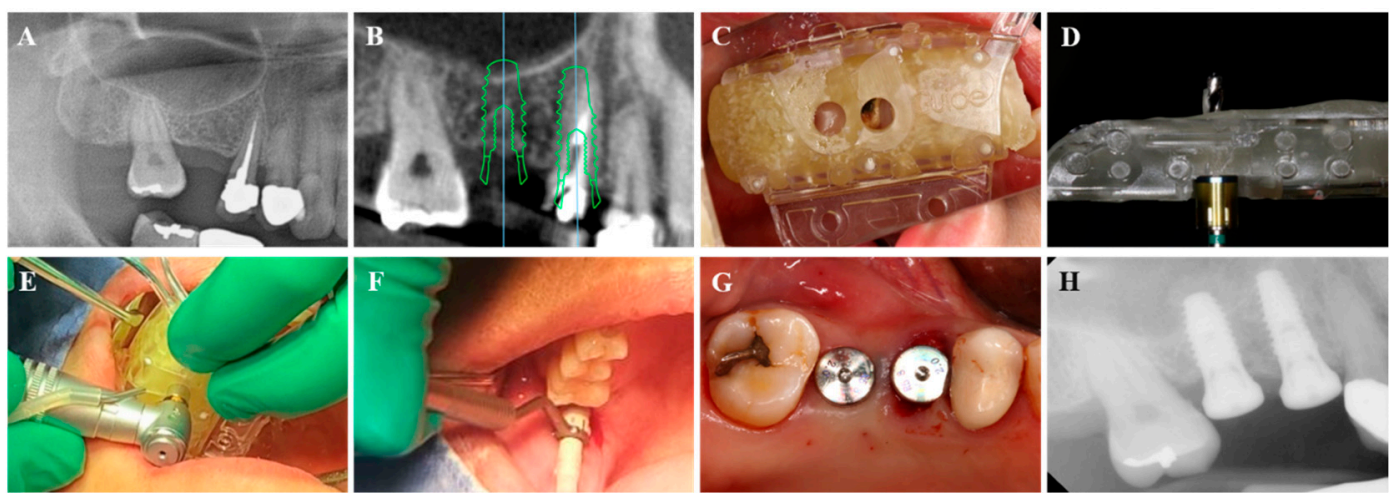

Figure 2. The simultaneous crestal sinus grafting procedure using the VARO guide. (A) A section of the pre-surgical panoramic radiograph showing the site of implantation. The upper right second premolar was vertically fractured; therefore, an immediate implant was planned. (B) Virtual planning of implants on upper right second premolar and molar regions on CBCT imaging. (C) The fabricated VARO guide was seated in the mouth after the extraction of the upper right second premolar. (D) The Sinus Drill with a stopper of the correct length was used for the initial entry into the maxillary sinus. (E) Sequential osteotomy was performed using the VARO guide. (F) A hydraulic tube was utilized for the elevation of the Schneiderian membrane. (G) Tissue-level implants were placed. (H) A periapical radiograph showing the typical "dome-shape" elevation following the crestal sinus graft.

Flapless surgery was performed using the VARO guide sinus kit (Neobiotech, Seoul, Korea). Infiltration anesthesia was performed at the surgical site using $2 \%$ lidocaine with 1:80,000 epinephrine. The thickness of the drill sleeve was determined so that the distance from the top of the drill sleeve to the alveolar bone crest was either 12 or $10.5 \mathrm{~mm}$, depending on the availability of the mouth opening. All the drills were designed with different lengths of adaptable stoppers so that full-depth drilling can be performed according to varying remaining ridge heights. A soft tissue punch, which is included in the system, was used to expose the alveolar bone at the drilling site. Using the Sinus Drill with a stopper of the correct length, the drilling was performed to just short of the sinus floor. Then, an S-Reamer, having a non-cutting tip, was used to elevate a thin lid of bone at the sinus floor into the sinus. The sinus membrane was checked for perforation using a depth gauge as well as the Valsalva maneuver. The sinus membrane was elevated using saline injected through the hydraulic syringe. A particulate bovine bone substitute (Bio-Oss; Geistlich Pharma AG, Wolhusen, Switzerland) was delivered via a bone carrier through the osteotomy site. If needed, widening of the osteotomy site was performed using the twist drill of the appropriate diameter. Finally, a tissue-level implant fixture (IT III, Neobiotech, Seoul, Korea) was placed with the VARO guide in situ. An optical impression was taken with a scan body connected to the fixture, and then the scan body was replaced with a healing abutment. Periapical radiographs of the placed implants were taken immediately and 3 months after surgery.

\subsection{Outcomes}

\subsubsection{Clinical Observation}

The surgical site was inspected for any signs of adverse reaction, such as bleeding, swelling, tenderness, and failure of osseointegration during the initial healing and followup periods. Then the wound was dressed on the day after surgery using hydrogen 
peroxide, and follow-up examinations were performed at 10 days, 4 weeks, and 3 months postoperatively.

\subsubsection{Radiographic Observation}

On the CBCT images, the remaining ridge height was measured around the virtually positioned implant. The remaining ridge height was defined as the height of the alveolar ridge at the lowest point of the curvature in the sinus floor contacting the implant fixture. After the surgery, the periapical radiographs were checked for any signs of membrane perforation, including the scattering of the graft material. The height of sinus membrane elevation was measured on the periapical radiographs immediately and 3 months after surgery.

\subsubsection{Accuracy of Implant Placement}

Superimposition of the Virtually Planned Implant and the Actually Placed Implant

The superimposition and measurement process of placement accuracy using the VARO guide are summarized in Figure 3.
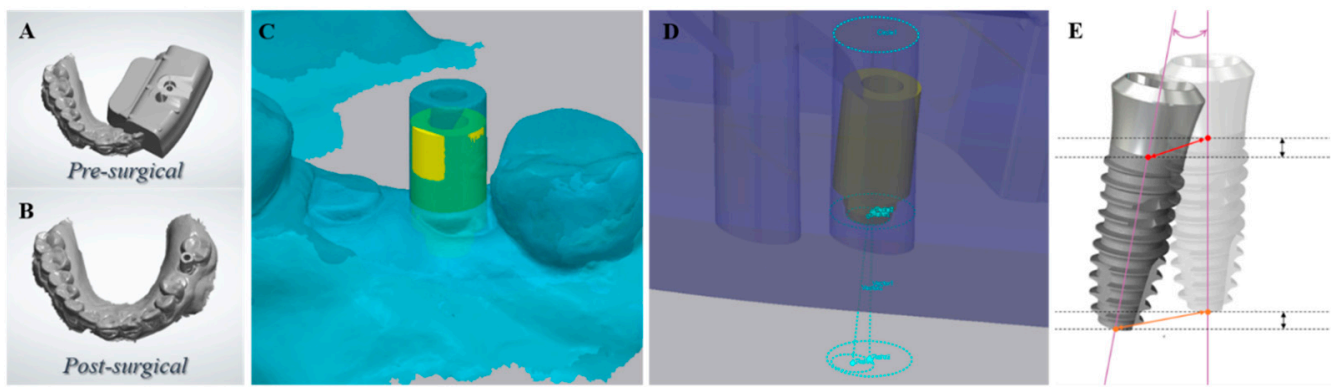

Figure 3. Measurement of the positional accuracy of the VARO guide surgery. (A) The pre-surgical plan data was uploaded to a dental CAD software. (B) The STL files from the postsurgical optical impression were also uploaded to the same software. (C) The data set was transferred to a $3 \mathrm{D}$ analysis software and superimposed using teeth as references. (D) The drilling hole from the pre-surgical data and the virtual abutment from the postsurgical data were used to "reverse engineer" the fixture positions. (E) The angular, vertical, and horizontal deviations were measured at implant apex and platform levels.

The pre-surgical plan data comprising the CBCT imaging and the guide design were extracted from the VG planning software (VARO Plan, Neobiotech, Seoul, Korea) and uploaded to a dental CAD software (exoCAD, exocad GmbH, Darmstadt, Germany). The STL files from the postsurgical optical impression were also uploaded to the same software, and the scan body was transformed into a virtual abutment. This data set containing the pre and postsurgical information was transferred to a 3D analysis software (Geomagic Verify, SculptCAD, Dallas, TX, USA), and by using teeth as the reference, the pre and postsurgical data were superimposed. The pre-planned fixture position was extrapolated from the position of the drilling hole and the postsurgical fixture position from the virtual abutment. This process of extrapolating the fixture position is known as "reverse engineering" and has been described in a previous study [11].

Parameters for Accuracy of Implant Placement

The measurements were taken by one examiner (Joo-Nyun Kim). Each parameter was measured twice, and then the mean of the two measurements was used.

- Vertical deviation (mm): the vertical distance between the planned and placed fixture apices.

- Angular deviation $\left(^{\circ}\right)$ : the angle between the axes of planned and placed fixtures.

- Horizontal platform deviation ( $\mathrm{mm}$ ): the horizontal distance between the planned and placed fixtures at the base of the transmucosal component of the tissue level implants. 
- Horizontal apex deviation ( $\mathrm{mm}$ ): the horizontal distance between the planned and placed fixture apices.

\subsubsection{Implant Stability}

Implant stability was measured immediately after implant placement and at the 3-month follow-up using a resonance frequency analysis device (RFA) (Osstell ISQ, Integration Diagnostics AB, Gothenburg, Sweden). RFA is a widely used analyzer that shows implant stability quotient (ISQ) values ranging from 1 to 100. ISQ values of 1-59 indicate low stability, 60-69 indicate medium stability, and 70 or higher indicate high stability.

\section{Results}

\subsection{Clinical Observation}

The detailed information of each site has been presented in Table 1.

Table 1. Demographic information of the surgical sites.

\begin{tabular}{ccccccc}
\hline Case & Sex & Age & $\begin{array}{c}\text { Implant } \\
\text { Placement } \\
\text { Site }\end{array}$ & $\begin{array}{c}\text { Size of Implant } \\
\text { Fixture } \\
\text { (Diameter } \times \\
\text { Length } \mathbf{m m})\end{array}$ & $\begin{array}{c}\text { Initial Torque/ } \\
\text { * Bone Quality/* } \\
\text { Bone Quantity }\end{array}$ & $\begin{array}{c}\text { Remaining } \\
\text { Ridge } \\
\text { Height } \\
\text { (mm) }\end{array}$ \\
\hline 1 & F & 76 & 26 & $5.0 \times 8.5$ & 40 N/D2/B & 3.6 \\
2 & F & 78 & 16 & $5.0 \times 10$ & $50 \mathrm{~N} / \mathrm{D} 2 / \mathrm{B}$ & 8.19 \\
3 & $\mathrm{~F}$ & 52 & 16 & $4.5 \times 8.5$ & $50 \mathrm{~N} / \mathrm{D} 2 / \mathrm{B}$ & 5.85 \\
4 & $\mathrm{~F}$ & 60 & 27 & $5.0 \times 10$ & $30 \mathrm{~N} / \mathrm{D} 3 / \mathrm{B}$ & 8.39 \\
\hline
\end{tabular}

ISQ; implant stability quotient. * Classification according to Lekholm and Zard.

Cases 1 and 4 were in the most-posterior single-tooth gap of the maxillary arch. Case 3 was the only case with an edentulous region of more than one tooth, having placed two consecutive implants. The default height from the top of the drill sleeve to the alveolar bone crest was $12 \mathrm{~mm}$. However, the length was reduced to $10.5 \mathrm{~mm}$ in Case 2 due to limited mouth opening. Wide implants having diameters of at least $4.5 \mathrm{~mm}$ were placed in all sites with the minimum placement torque of $30 \mathrm{~N}$.

No tearing of the sinus membrane was evident during surgery and in the postoperative radiographs. All of the treated sites healed uneventfully. Prosthetic treatment had been completed following the 3-month visit for all cases.

\subsection{Radiographic Observation}

The periapical radiographs of each case taken at baseline and 3 months postoperative have been shown in Figure 4. The mean and standard deviation of the remaining ridge heights of the four cases was $6.68 \pm 1.99 \mathrm{~mm}$. Case 1 had the shortest remaining ridge height of $3.6 \mathrm{~mm}$, and Case 4 had the tallest remaining ridge height of $8.39 \mathrm{~mm}$. The periapical radiographs taken after surgery revealed the typical "dome-shaped' elevation of the sinus membrane without signs of membrane tear. The mean amount of sinus membrane elevation in all cases was $5.29 \pm 1.41 \mathrm{~mm}$ immediately after surgery and $5.27 \pm 1.36 \mathrm{~mm}$ at 3 months after surgery. 


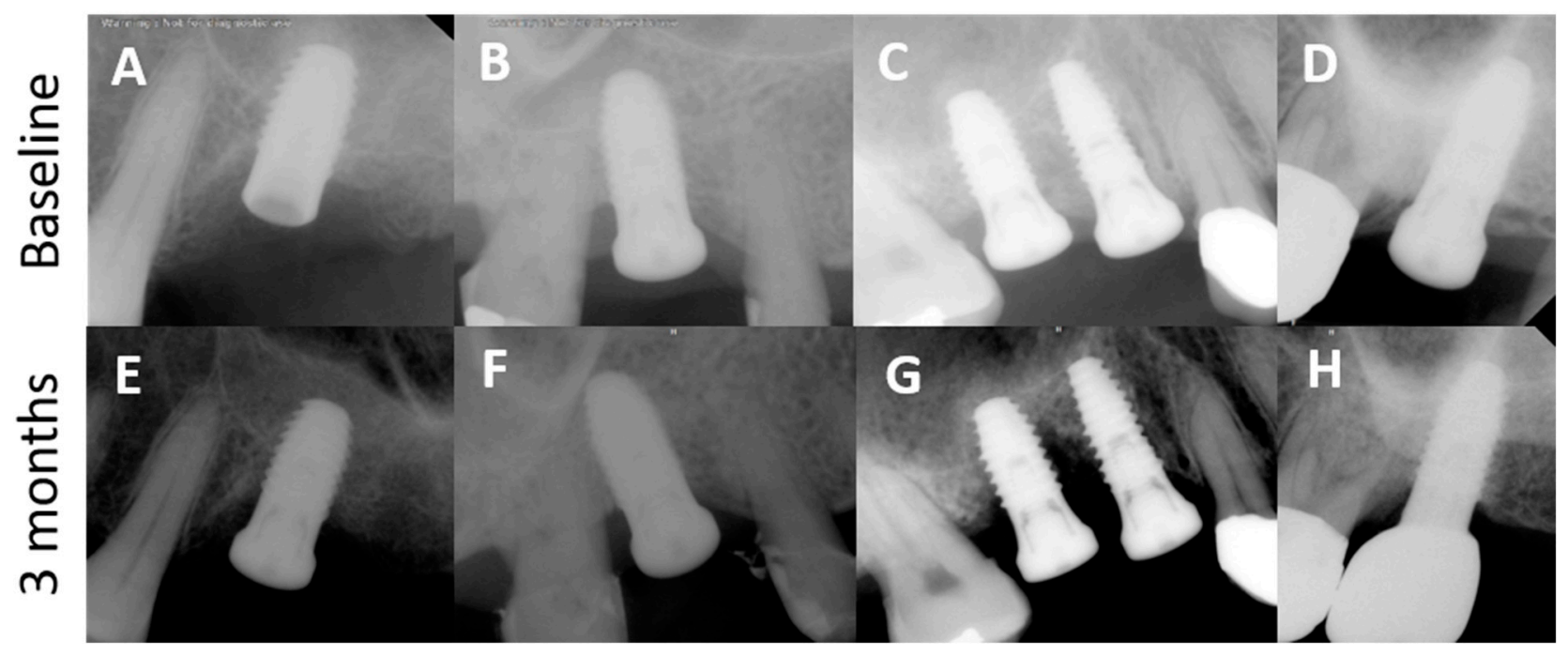

Figure 4. Periapical radiographs taken at baseline and 3 months. (A, B, C, and D) represent Cases 1, 2, 3, and 4, respectively, at baseline. (E, F, G, and $\mathbf{H}$ ) represent cases 1, 2, 3, and 4, respectively, at 3 months postoperative.

\subsection{Accuracy of Implant Placement Using the VARO Guide}

The measurements for the accuracy of implant placement have been shown in Table 2.

Table 2. Parameters showing deviation between virtually planned and actually placed implants.

\begin{tabular}{ccccc}
\hline Case & Hp $(\mathbf{m m})$ & Ha $(\mathbf{m m})$ & $\left.\mathbf{A ~} \mathbf{(}^{\circ}\right)$ & $\mathbf{V}(\mathbf{m m})$ \\
\hline 1 & 1.85 & 2.82 & 6.39 & 0.96 \\
2 & 1.62 & 2.18 & 5.45 & 1.53 \\
3 & 0.87 & 1.41 & 4.71 & 0.54 \\
4 & 0.75 & 0.97 & 2.49 & 0.31 \\
Mean \pm SD & $1.27 \pm 0.47$ & $1.85 \pm 0.71$ & $4.76 \pm 1.44$ & $0.84 \pm 0.46$ \\
Median (Min, Max) & $1.25(0.75,1.85)$ & $1.80(0.97,2.82)$ & $5.08(2.49,6.39)$ & $0.75(0.31,1.53)$ \\
\hline
\end{tabular}

Hp: horizontal deviation at implant platform (mm). Ha: horizontal deviation at implant apex (mm). A: Angular deviation. V: Vertical deviation at the apex $(\mathrm{mm})$.

The total of four implants placed using the VARO guide demonstrated mean deviations of $1.27 \pm 0.47 \mathrm{~mm}$ horizontally at the platform, $1.85 \pm 0.71 \mathrm{~mm}$ horizontally at apex, $4.76 \pm 1.44^{\circ}$ in angle, and $0.84 \pm 0.46 \mathrm{~mm}$ vertically at the apex. Case 4 , having the tallest height of the remaining ridge, exhibited the most accurate implant placement in regards to all parameters. Case 1, having the shortest remaining ridge height, was the least accurate in the horizontal and angular aspects. Case 2, placed in the upper right first molar region using the reduced sleeve length of $10.5 \mathrm{~mm}$, was the least accurate in the vertical aspect. In terms of the errors in angular and horizontal aspects, there was a trend of reduction from Case 1 to Case 4.

\subsection{Implant Stability}

The results from implant stability measurements are shown in Figure 5. 


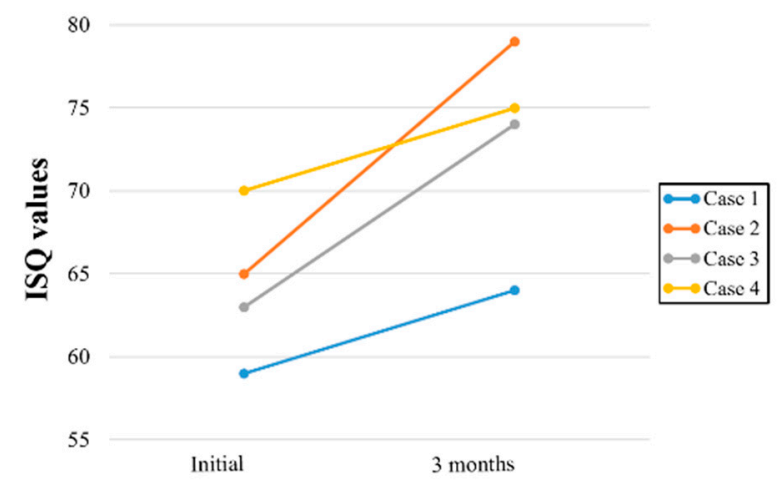

Figure 5. Implant stability quotient (ISQ) values measured using the resonance frequency analysis. Case 1 having the least remaining vertical ridge height showed the least initial stability, and vice versa for Case 4 .

All implants were placed with sufficient initial torque and stability for the connection of healing abutments. The initial placement torque ranged from 30 to $50 \mathrm{~N}$ in all cases. ISQ values showed a pattern of increase from the initial to the 3-month postoperative period. Case 1 exhibited the lowest stability at immediate and 3-month postoperative, represented by ISQ values of 59 and 64 , respectively. These values indicate the borderline between low and medium stability of the implant fixture. Medium to high stability was achieved in the other cases. Case 4 exhibited the highest initial stability represented by ISQ values of 70 . All of the ISQ values were acceptable for the application of conventional loading protocol in this study.

\section{Discussion}

The VARO guide is a novel, fully-guided implant placement system characterized by the in-house, model-free, and zero-setup approaches. The present pilot study was performed to evaluate the accuracy and feasibility of the VARO guide system in the atrophic posterior maxilla for fully-guided flapless implant placement with simultaneous crestal sinus graft. From the four implants that had been placed using the VARO guide, the main findings of the study were: (i) the VARO guided surgery exhibited a range of deviation from the planned position of around $1 \mathrm{~mm}$, (ii) simultaneous crestal sinus graft can be performed safely and without complication, and (iii) good stability was achieved for the placed implants at the initial and late stages.

According to a recent meta-analysis of 669 implants placed in partially edentulous areas, the mean global deviation values were $2.68^{\circ}$ in angle; $1.03 \mathrm{~mm}$ coronally; $1.33 \mathrm{~mm}$ apically; and $0.59 \mathrm{~mm}$ vertically [12]. In the current study, the deviation values were $4.76^{\circ}$ in angle, $1.27 \mathrm{~mm}$ horizontally at the platform, $1.85 \mathrm{~mm}$ horizontally at apex, $0.84 \mathrm{~mm}$ vertically at the apex. The deviation values revealed here were slightly larger than that of the meta-analysis. This could be due to the fact that these implants were placed in a challenging situation of the atrophic posterior ridge, where there is a lack of bone support at the apical region of the implant fixture. Furthermore, all of the cases in this study were in the posterior region, the majority of them in the most posterior tooth of the arch, whereas the data from the meta-analysis was a combination of data from the anterior region. There is evidence in the literature that guided surgery in the anterior region produces more accurate results compared to the posterior since implant placement procedures in the molar region are more often faced with difficulties related to access and vision [13]. For evaluation of the guide suitability for clinical application, two criteria can be considered: (i) the possibility to deliver the immediate provisional prosthesis by passive fit (i.e., without chairside correction), and (ii) the safety margin deduced from the deviation data of the surgical guide. The recorded values in the present study were greater than the prosthetic requirement for the passive fit of immediate provisional restorations, which has been reported to be around 0.1-0.15 mm [14-16]. Achieving such a high degree of precision seems difficult 
despite the current technological advances. Concerning safety, the vertical deviation at the apex is probably the most relevant outcome as it affects the proximity to vital anatomical structures [12]. With that being said, the vertical deviation of less than $1 \mathrm{~mm}$ demonstrated using the VARO guide could be considered "safe" in regards to preventing injuries to the inferior mandibular nerve and perforation of the Schneiderian membrane.

Case 4, which was placed in the most-posterior single tooth region at the upper second molar, was the most accurate in all aspects among the four cases. This case also showed the greatest remaining ridge height of $8.39 \mathrm{~mm}$. Case 2 had a comparable remaining ridge height of $8.19 \mathrm{~mm}$; however, it was the least accurate in the vertical aspect. This was owed to the limited mouth opening in Case 2 and the subsequent difficulty in maneuvering the drill. Design solutions of the surgical guide to overcome the lack of vertical dimension include opening the buccal side of the drill sleeve to allow drill entry and reducing the sleeve length. Whether the buccal opening of the drill sleeve affects accuracy has been investigated previously [13]. In that study, the use of the open sleeve design resulted in less accurate implant placement in the posterior region. In another study that investigated the effect of drilling distance, sleeve length, and sleeve height, it has been shown that the length of the sleeve had no influence on accuracy, but rather the drilling distance below the sleeve had a significant effect on the accuracy of implant placement [17]. The VARO guide has adopted the latter design of reduced sleeve length rather than the open-sleeve design, which should produce greater accuracy according to these indications in the literature.

The four cases included in this study exhibited a variation of deviation values. Case 1 showed the greatest deviation in the angle and horizontal aspects at the platform and apex. This result could be owed to Case 1 having the least remaining ridge height among all of the cases. In contrast, Case 4, having the tallest remaining ridge height, revealed the most accurate result. There was also a correlation between the remaining ridge height and the initial stability of the implants, as Case 1 was the least stable and Case 4 was the most stable. Taking this into consideration, it could be deduced that shorter ridge height can lead to less implant stability and less accurate implant placement.

Another possible explanation for the variation among the data might be the influence of the learning curve. The four cases were performed in chronological order; therefore, the skill of the surgeon at using the VARO guide would have improved after each case. This was evident in the data, as there was a trend of decrease in error from Case 1 through to Case 4. However, due to the small number of subjects in this pilot study, it is difficult to draw a conclusion based on statistics. In the authors' knowledge, there has not been a prospective trial up-to-date on the accuracy of guided implant placement with simultaneous crestal sinus graft. It is necessary, therefore, to perform a randomized controlled trial to verify the effect of the simultaneous flapless approach on the accuracy of guided implant placement.

It is also possible that other sources of error might have contributed toward the deviation data in this study. Inaccuracies in VARO-guided surgery can be attributed to factors that are intrinsic and extrinsic to the surgical guide. Intrinsic errors can arise from the inaccuracy of the composite resin impression using the pre-guide and incorrect seating of the pre-guide during CBCT taking. Polymerization shrinkage is an inherent drawback of resin composite [18], and since the inner surfaces of the pre-guide had been cured extraorally, shrinkage of the fitting surfaces can be a possibility. Extrinsic errors can result from the movement of the VARO guide during implant placement and the experience of the surgeon in the VARO guide protocol. In regards to the latter, however, a recent in vitro study has reported similar outcomes in terms of time and accuracy when VARO guide surgery was performed by expert and novice users [11]. In the same study, when the VARO guide was compared to a 3D-printed surgical guide, the VARO guide required less time to prepare, although the surgical time was similar.

Since the evolution of implant dentistry toward minimally invasive procedures, flapless surgery has been shown to provide many benefits. These include preservation of blood circulation and soft tissue architecture [19], prevention of marginal bone loss by avoiding bone denudation during flap elevation [20-22], and considerably less postoperative mor- 
bidity and discomfort in terms of the patient-reported outcome [23-25]. However, it can be disadvantageous to perform flapless surgery freehanded as the true outline of the underlying bone cannot be detected by palpation alone [26]. Furthermore, in the vertically atrophic ridge, as in this study, accurate implant placement may seem like a challenging task. In the VARO guide sinus kit, a soft tissue punch is included, and it has become possible to access the sinus floor without raising a flap and place the implant fixture according to the virtual plan. The advantage of this digital technique is that even when the sinus floor is curved, the exact point of entry can be predicted and accurately accessed using the surgical guide. Furthermore, elevating the sinus membrane using hydraulic pressure can help to prevent the perforation of the sinus membrane and also detach the membrane from the sinus floor more evenly around the implant fixture than the osteotome technique. In this pilot study, all four cases were performed successfully without any complications, such as perforation of the sinus membrane, and all implants were placed with stable initial torque of at least $30 \mathrm{~N}$. In this regard, it could be proposed that the simultaneous crestal sinus graft using the VARO guide sinus kit is a feasible technique when faced with remaining ridge heights of 4 to $8 \mathrm{~mm}$.

\section{Conclusions}

In summary, fully-guided implant placement with simultaneous crestal sinus graft using the VARO guide could be a safe and feasible method with acceptable accuracy. The limitation of the present pilot study was the paucity of data. Randomized controlled trials comparing the VARO guide to a conventional or 3D-printed surgical guide should be performed in the future to validate its clinical performance in terms of surgical time, accuracy, and patient-reported outcomes.

Author Contributions: Conceptualization, J.-K.C.; methodology, J.-K.C.; software, J.-N.K.; validation, J.-K.C. and J.-S.L.; formal analysis, J.-N.K.; investigation, J.-Y.L. and J.-Y.P.; resources, J.-K.C.; data curation, J.-Y.L.; writing — original draft preparation, J.-Y.P. and J.-Y.L.; writing—review and editing, J.-K.C., J.-W.P., U.-W.J. and S.-H.C.; visualization, J.-K.C. and U.-W.J.; supervision, J.-S.L., S.-H.C., U.-W.J. and J.-W.P.; project administration, J.-K.C.; funding acquisition, J.-K.C. All authors have read and agreed to the published version of the manuscript.

Funding: This work was supported by the Korea Medical Device Development Fund grant funded by the Korea government (the Ministry of Science and ICT, the Ministry of Trade, Industry and Energy, the Ministry of Health \& Welfare, the Ministry of Food and Drug Safety) (Project Number: KMDF_PR_20200901_0257).

Institutional Review Board Statement: This study was performed according to the Declaration of Helsinki. The trial received ethical approval from the Institutional Review Board of the Yonsei University Dental Hospital (2-2020-0053), and it was registered with the Clinical Research Information Service of the National Research Institute of Health in the Republic of Korea (KCT0005465).

Informed Consent Statement: Informed consent was obtained from all subjects involved in the study. Written informed consent has been obtained from the patients to publish this paper.

Data Availability Statement: The data presented in this study are available on request from the corresponding author.

Conflicts of Interest: The authors declare no conflict of interest.

\section{References}

1. Testori, T.; Weinstein, T.; Scutellà, F.; Wang, H.-L.; Zucchelli, G. Implant placement in the esthetic area: Criteria for positioning single and multiple implants. Periodontology 2000 2018, 77, 176-196. [CrossRef]

2. D'Haese, J.; Ackhurst, J.; Wismeijer, D.; De Bruyn, H.; Tahmaseb, A. Current state of the art of computer-guided implant surgery. Periodontol 2000 2017, 73, 121-133. [CrossRef] [PubMed]

3. Romanos, G.E.; Delgado-Ruiz, R.; Sculean, A. Concepts for prevention of complications in implant therapy. Periodontology 2000 2019, 81, 7-17. [CrossRef]

4. Choi, W.; Nguyen, B.C.; Doan, A.; Girod, S.; Gaudilliere, B.; Gaudilliere, D. Freehand Versus Guided Surgery: Factors Influencing Accuracy of Dental Implant Placement. Implant Dent. 2017, 26, 500-509. [CrossRef] 
5. Smitkarn, P.; Subbalekha, K.; Mattheos, N.; Pimkhaokham, A. The accuracy of single-tooth implants placed using fully digitalguided surgery and freehand implant surgery. J. Clin. Periodontol. 2019, 46, 949-957. [CrossRef] [PubMed]

6. Park, J.Y.; Song, Y.W.; Park, S.H.; Kim, J.H.; Park, J.M.; Lee, J.S. Clinical factors influencing implant positioning by guided surgery using a nonmetal sleeve template in the partially edentulous ridge: Multiple regression analysis of a prospective cohort. Clin. Oral Implants Res. 2020, 31, 1187-1198. [CrossRef]

7. Velasco-Ortega, E.; Jiménez-Guerra, A.; Ortiz-Garcia, I.; Moreno-Muñoz, J.; Núñez-Márquez, E.; Cabanillas-Balsera, D.; LópezLópez, J.; Monsalve-Guil, L. Immediate Loading of Implants Placed by Guided Surgery in Geriatric Edentulous Mandible Patients. Int. J. Environ. Res. Public Health 2021, 18, 4125. [CrossRef]

8. Kan, J.Y.K.; Rungcharassaeng, K.; Deflorian, M.; Weinstein, T.; Wang, H.-L.; Testori, T. Immediate implant placement and provisionalization of maxillary anterior single implants. Periodontology 2000 2018, 77, 197-212. [CrossRef]

9. Marra, R.; Acocella, A.; Rispoli, A.; Sacco, R.; Ganz, S.D.; Blasi, A. Full-mouth rehabilitation with immediate loading of implants inserted with computer-guided flap-less surgery: A 3-year multicenter clinical evaluation with oral health impact profile. Implant Dent. 2013, 22, 444-452. [CrossRef] [PubMed]

10. Pozzi, A.; Tallarico, M.; Marchetti, M.; Scarfò, B.; Esposito, M. Computer-guided versus free-hand placement of immediately loaded dental implants: 1-year post-loading results of a multicentre randomised controlled trial. Eur. J. Oral Implantol. 2014, 7, 229-242. [PubMed]

11. Song, Y.; Kim, J.; Kim, J.-H.; Park, J.-M.; Jung, U.-W.; Cha, J.-K. Accuracy of Dental Implant Placement by a Novel In-House Model-Free and Zero-Setup Fully Guided Surgical Template Made of a Light-Cured Composite Resin (VARO Guide ${ }^{\circledR}$ ): A Comparative In Vitro Study. Materials 2021, 14, 4023. [CrossRef]

12. Siqueira, R.; Chen, Z.; Galli, M.; Saleh, I.; Wang, H.; Chan, H. Does a fully digital workflow improve the accuracy of computerassisted implant surgery in partially edentulous patients? A systematic review of clinical trials. Clin. Implant. Dent. Relat. Res. 2020, 22, 660-671. [CrossRef]

13. Tallarico, M.; Kim, Y.-J.; Cocchi, F.; Martinolli, M.; Meloni, S.M. Accuracy of newly developed sleeve-designed templates for insertion of dental implants: A prospective multicenters clinical trial. Clin. Implant. Dent. Relat. Res. 2019, 21, 108-113. [CrossRef]

14. Castillo-De-Oyague, R.; Sanchez-Turrion, A.; Lopez-Lozano, J.; Albaladejo, A.; Torres-Lagares, D.; Montero, J.; Suarez-Garcia, M Vertical misfit of laser-sintered and vacuum-cast implant-supported crown copings luted with definitive and temporary luting agents. Medicina Oral Patología Oral y Cirugia Bucal 2012, 17, e610-e617. [CrossRef]

15. Castillo-Oyagüe, R.; Lynch, C.D.; Turrión, A.S.; López-Lozano, J.F.; Torres-Lagares, D.; Suárez-García, M.-J. Misfit and microleakage of implant-supported crown copings obtained by laser sintering and casting techniques, luted with glass-ionomer, resin cements and acrylic/urethane-based agents. J. Dent. 2013, 41, 90-96. [CrossRef]

16. Pereira, L.M.S.; Sordi, M.B.; Magini, R.S.; Duarte, A.R.C.; Souza, J.C. Abutment misfit in implant-supported prostheses manufactured by casting technique: An integrative review. Eur. J. Dent. 2017, 11, 553-558. [CrossRef]

17. El Kholy, K.; Janner, S.F.M.; Schimmel, M.; Buser, D. The influence of guided sleeve height, drilling distance, and drilling key length on the accuracy of static Computer-Assisted Implant Surgery. Clin. Implant. Dent. Relat. Res. 2019, 21, 101-107. [CrossRef]

18. Kaisarly, D.; Gezawi, M.E. Polymerization shrinkage assessment of dental resin composites: A literature review. Odontology 2016, 104, 257-270. [CrossRef] [PubMed]

19. Sclar, A.G. Guidelines for Flapless Surgery. J. Oral Maxillofac. Surg. 2007, 65 (Suppl. 1), 20-32. [CrossRef]

20. Staffileno, H. Significant Differences and Advantages Between the Full Thickness and Split Thickness Flaps. J. Periodontol. 1974, 45, 421-425. [CrossRef]

21. Becker, W.; Goldstein, M.; Becker, B.E.; Sennerby, L. Minimally Invasive Flapless Implant Surgery: A Prospective Multicenter Study. Clin. Implant. Dent. Relat. Res. 2005, 7 (Suppl. 1), s21-s27. [CrossRef]

22. Rocci, A.; Dds, M.M.; Gottlow, J. Immediate Loading in the Maxilla Using Flapless Surgery, Implants Placed in Predetermined Positions, and Prefabricated Provisional Restorations: A Retrospective 3-Year Clinical Study. Clin. Implant. Dent. Relat. Res. 2003, 5 (Suppl. 1), 29-36. [CrossRef]

23. Becker, W.; Goldstein, M.; Becker, B.E.; Sennerby, L.; Kois, D.; Hujoel, P. Minimally invasive flapless implant placement: Follow-up results from a multicenter study. J. Periodontol. 2009, 80, 347-352. [CrossRef]

24. Fortin, T.; Bosson, J.L.; Isidori, M.; Blanchet, E. Effect of flapless surgery on pain experienced in implant placement using an image-guided system. Int. J. Oral Maxillofac. Implant. 2006, 21, 298-304.

25. Oh, T.-J.; Shotwell, J.L.; Billy, E.J.; Wang, H.-L. Effect of flapless implant surgery on soft tissue profile: A randomized controlled clinical trial. J. Periodontol. 2006, 77, 874-882. [CrossRef]

26. Chen, Z.; Li, J.; Sinjab, K.; Mendonca, G.; Yu, H.; Wang, H.-L. Accuracy of flapless immediate implant placement in anterior maxilla using computer-assisted versus freehand surgery: A cadaver study. Clin. Oral Implant. Res. 2018, 29, 1186-1194. [CrossRef] 